\title{
Freezing of gait and white matter changes: a tract-based spatial statistics study
}

\author{
Kazumi Iseki ${ }^{1,2,3,4^{*}}$, Hidenao Fukuyama', Naoya Oishi ${ }^{1}$, Hidekazu Tomimoto ${ }^{5}$, Yoshinobu Otsuka ${ }^{1}$, \\ Manabu Nankaku ${ }^{6}$, David Benninger ${ }^{2}$, Mark Hallett ${ }^{2}$ and Takashi Hanakawa ${ }^{1,7,8}$
}

\begin{abstract}
Background: We hypothesized that the integrity of white matter might be related to the severity of freezing of gait in age-related white matter changes.

Methods: Twenty subjects exhibiting excessive hyperintensities in the periventricular and deep white matter were recruited. The subjects underwent the Freezing of Gait Questionnaire, computerized gait analyses, and diffusion tensor magnetic resonance imaging. Images of axial, radial and mean diffusivity, and fractional anisotropy were calculated as indices of white matter integrity and analyzed with tract-based spatial statistics.

Results: The fractional anisotropy, mean, axial and radial diffusivity averaged across the whole white matter structure were all significantly correlated with Freezing of Gait Questionnaire scores. Regionally, a negative correlation between Freezing of Gait Questionnaire scores and fractional anisotropy was found in the left superior longitudinal fasciculus beneath the left premotor cortex, right corpus callosum, and left cerebral peduncle. The scores of the Freezing of Gait Questionnaire were positively correlated with mean diffusivity in the left corona radiata and right corpus callosum, and with both axial and radial diffusivity in the left corona radiata. The white matter integrity in these tracts (except the corpus callosum) showed no correlation with cognitive or other gait measures, supporting the specificity of those abnormalities to freezing of gait.
\end{abstract}

Conclusion: Divergent pathological lesions involved neural circuits composed of the cerebral cortex, basal ganglia and brainstem, suggesting that freezing of gait has a multifactorial nature.

Keywords: Age-related white matter change (ARWMC), Diffusion tensor imaging (DTI), Disconnection, Freezing of gait (FOG), Tract-based spatial statistics (TBSS), Vascular Parkinsonism

\section{Background}

Freezing of gait (FOG) is a characteristic symptom observed in some patients with gait disorders. FOG is defined as an "inability to generate effective stepping movement" [1]. It is often observed in patients with idiopathic Parkinson's disease (PD), and is even more prevalent in patients with atypical Parkinsonism (including vascular Parkinsonism) than in those with PD [2]. FOG can be seen in age-related white matter changes (ARWMC), which refer to the neuroradiological

\footnotetext{
* Correspondence: kazumi.iseki@gmail.com

${ }^{1}$ Human Brain Research Center, Kyoto University Graduate School of Medicine, 54 Kawahara-cho, Shogoin, Sakyo-ku, Kyoto 606-8507, Japan

${ }^{2}$ Human Motor Control Section, Medical Neurology Branch, National Institute of Neurological Disorders and Stroke, National Institutes of Health, Bethesda, MD, USA

Full list of author information is available at the end of the article
}

state of diffusely extended white matter lesions in elderly subjects [3-5].

The pathophysiology of FOG remains unclear [6]. The most reliable measure of the severity in FOG to date is the total score on the Freezing of Gait Questionnaire (FOGQ) [7]. Following recent development, white matter (WM) integrity can now be evaluated using diffusion tensor imaging (DTI). A directional bias of water diffusion can be quantified by measuring fractional anisotropy (FA), while mean diffusivity (MD) indicates the degree of diffusion [8].

Analysis of DTI can directly test whether the corticosubcortical disconnection of WM tracts is responsible for gait disturbance as suggested by previous studies on gait disturbance in ARWMC [9,10]. Tract-based spatial statistics (TBSS) analysis of DTI data was recently 
developed as an automatic, hypothesis-free and precise method for the assessment of integrity of the WM [11]. Here we investigated the degree of integrity and directionality of the WM tracts in ARWMC patients, by means of TBSS analysis of DTI in combination with FOGQ scores.

\section{Methods \\ Subjects}

Twenty subjects with ARWMC were enrolled at the Neurology Clinic of Kyoto University Hospital. The inclusion criteria were: (1) aged between 65 and 84 years old, and (2) $\mathrm{T}_{2}$-weighted magnetic resonance images (MRI) revealing both irregular periventricular hyperintensities extending into the deep WM (Fazekas' PVH 3) and confluent hyperintensities in the deep WM (Fazekas' DWMH 3), diffusely involving at least bilateral frontosubcortical areas and not confined to a single vascular territory [12]. Exclusion criteria were: (1) a history of acute stroke in the previous 3 months, (2) neurological and neuroradiological findings or history of surgery which suggests complication of other neurological or orthopedic diseases affecting gait including possible idiopathic normal pressure hydrocephalus (NPH). Specifically, an experienced neurologist first selected subjects solely according to the neuroimaging findings in the neurology clinic. The subjects whose diagnoses were other than ARWMC were excluded afterwards according to the exclusion criteria. The research protocol was approved by the ethics committee of Kyoto University Graduate School and Faculty of Medicine, and written informed consent was obtained from each subject.

\section{Assessment of FOGQ and gait performance}

All subjects completed the FOGQ [7], and underwent neurological evaluation by a board-certified neurologist (K.I.). While subjects walked at their own pace repeatedly, the presence of FOG was checked, with special attention being paid to their gait initiation and turning. Moreover, they were assessed with a threedimensional (3D) locomotion analysis system (GATALITS-60, Sumitomo Metal Inc., Osaka, Japan). Velocity, cadence, stride length (SL), stride width (SW), gait cycle (GC), and the ratios of double support time per GC (DST/GC) were measured. The correlations between the gait-related parameters and the sum of FOGQ scores were tested (Spearman's test). Additionally, the total score of FOGQ was compared between the FOG positive and FOG negative groups as classified by the clinical observation (Mann-Whitney $U$ test). Findings were considered significant at the level of $P<0.05$.

\section{Assessment of cognitive status}

Mini-mental state examination (MMSE) scores were obtained. They were not intended to provide a precise evaluation of cognitive impairment, as our main focus was the analysis of FOG. We conducted Spearman's tests to test the correlations between FOGQ and MMSE scores. Findings were considered significant at the level of $P<0.05$.

\section{Image data acquisition}

DTI, $\mathrm{T}_{1}$ and $\mathrm{T}_{2}$-weighted images were acquired with a 3Tesla MRI scanner (Trio; Siemens, Erlangen, Germany). DTI data were obtained using a single-shot, spinecho, echo-planar imaging sequence applying motion probing gradient pulses to 12 non-colinear axes with $\mathrm{b}=700 \mathrm{~s} / \mathrm{mm}^{2}$. To enhance the signal-to-noise ratio, imaging was repeated four times.

A magnetization-prepared rapid gradient echo (MPRAGE) sequence was used for anatomical $\mathrm{T}_{1}$-weighted volume data acquisition. $\mathrm{T}_{2}$-weighted images were acquired with a turbo spin-echo sequence.

\section{Data analysis}

The DTI data were pre-processed using DTI-Fit software included in the Functional MRI of the Brain (FMRIB) Diffusion Toolbox (FDT) (FSL4.1, http://www. fmrib.ox.ac.uk/fsl). We computed FA images and diffusivity images representing axial diffusivity $\left(\mathrm{AD}, \lambda_{1}\right)$ and radial diffusivity ( $\left.\mathrm{RD}, \lambda_{2}+\lambda_{3} / 2\right)$ to the principal direction [13], and also mean diffusivity (MD). Representative $\mathrm{T}_{2^{-}}$ weighted images demonstrating $\mathrm{PVH}$ and DWMH, and maps of diffusion measures, both of which were taken from the same subject are shown in Figure 1.

First, we tested the relationship between the FOGQ score and the global changes of the diffusion measures reflecting different aspects of WM integrity (global correlation analysis). We hypothesized that FA would be decreased in accord with the severity of FOG (reflected by greater FOGQ scores) and that there would be a negative correlation between FOGQ and FA. As for the parameters representing diffusivity, MD is thought to reflect overall WM disruption similarly to FA, while RD and $\mathrm{AD}$ are thought to be useful as an index of myelin integrity and axonal integrity, respectively [13-15]. The most commonly reported pattern in previous studies is an increase in $\mathrm{RD}, \mathrm{AD}$, and $\mathrm{MD}$ in accord with the severity of age-related white matter damage [16]. We hence hypothesized that those diffusivity measures would be increased in relation to the severity of FOG and that there would be a positive correlation between FOGQ score and the three diffusivity parameters (MD, RD and $A D)$. The correlation of FOGQ scores with each of FA, MD, $\mathrm{AD}$ and $\mathrm{RD}$ in the mean WM skeleton was tested using Spearman's rank correlation.

To explore possible correlation between brain atrophy and FOGQ scores, the Structural Image Evaluation using Normalization of Atrophy (SIENAX) method [17] was then applied to the $T_{1}$-weighted image for each 


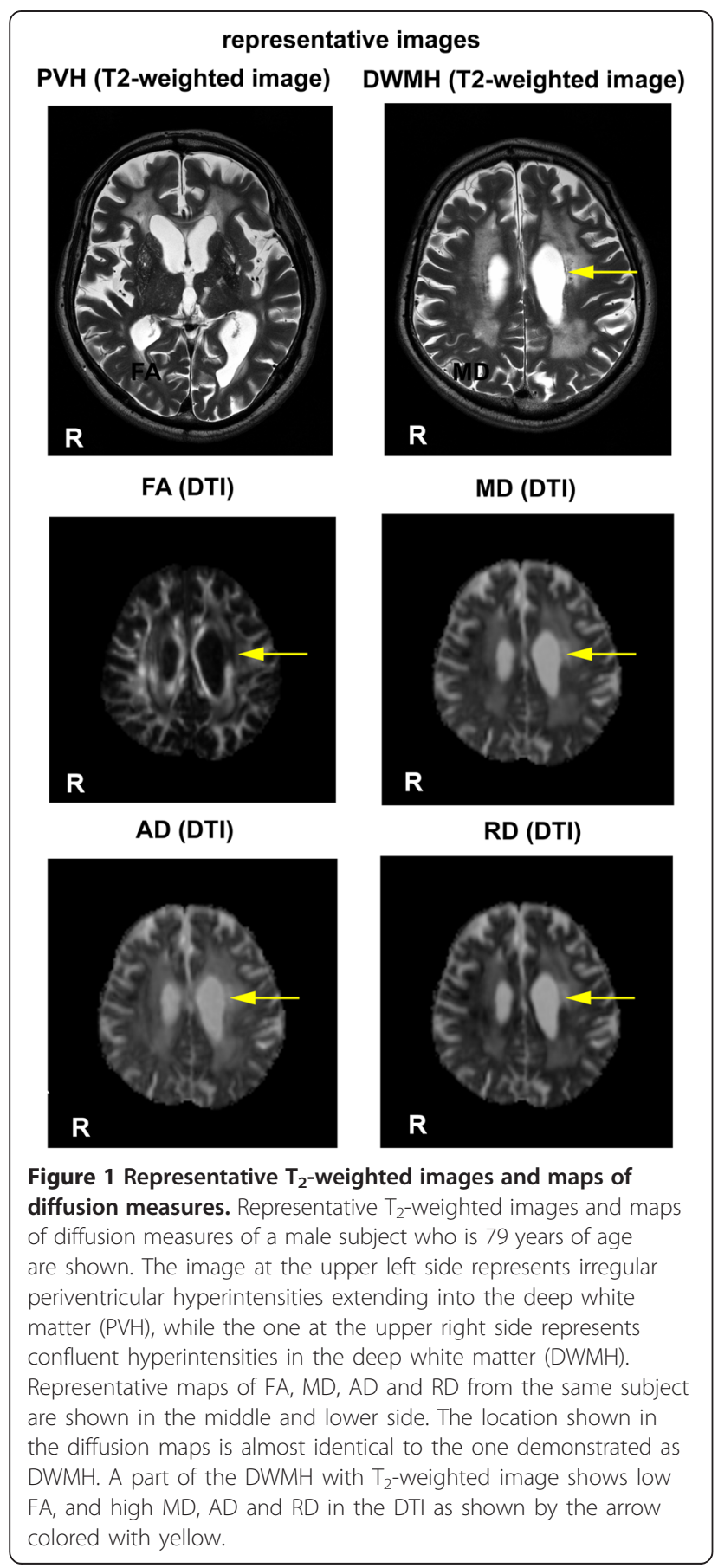

subject to estimate the total, gray and white matter brain volumes.

Second, we tested regional correlations between the FOGQ score and the image representing each of the diffusion parameters (FA, MD, AD, and RD) on a voxel-byvoxel basis using the TBSS software. For the regional correlation analysis, we also speculated that the FOGQ score would be negatively correlated with FA values, and positively correlated with $\mathrm{MD}, \mathrm{AD}$ and $\mathrm{RD}$ values.
Statistical inference was obtained using a non-parametric method called Threshold-Free Cluster Enhancement (TFCE) with 5,000 permutations [18]. Changes were considered significant at the level of $P<0.05$, which was fully corrected for multiple comparisons. To test the specific relation of FOGQ scores with the diffusion measures identified in the regional correlation analysis, a confirmatory multiple linear regression analysis was applied. We computed the average value of $F A, M D, A D$ and $R D$ in each cluster, which showed significant regional correlation with FOGQ score as a sole dependent variable. The FOGQ scores, MMSE scores, walking velocity, cadence, SL, SW, GC and DST/GC were used as independent variables. Testing the multicollinearity that affects the result of the analysis is considered to be important when employing the multivariate analysis. Multicollinearity between these independent variables was tested by calculating the variance inflation factor (VIF), which is widely used for this purpose. If the VIF reveals specific variables to be unacceptable in the model, it is advised to exclude those specific variables. A well-accepted cutoff value of VIF is $10[19,20]$. The result was considered significant at the level of $P<0.05$.

\section{Results}

\section{Behavioral parameters}

Clinical assessment of the subjects, including the FOGQ scores and the presence of FOG, is shown in Table 1. The FOGQ scores (mean \pm S.D.; $4.2 \pm 5.0$ ) were significantly correlated with the DST/GC $(0.31 \pm 0.074$, $r=0.63, P=0.003)$, walking velocity $(0.98 \pm 0.30, r=-0.79$, $P<0.001)$ and SL $(0.96 \pm 0.25, r=-0.74, P<0.001)$ (Table 2). Furthermore, there was a significant difference in FOGQ scores between subjects who were judged to exhibit FOG $(9.2 \pm 5.7, \mathrm{n}=6)$ and those who were not $(2.5 \pm 2.7, \mathrm{n}=14)$ $(P=0.003$, Mann-Whitney $U$ test). In addition, the FOGQ and the MMSE scores $(28 \pm 1.4)$ also showed a significant correlation $(r=-0.62, P=0.003$; Table 2$)$.

\section{Correlation of FOG with global changes in brain volumes and DTI measures}

The assessment of brain atrophy with SIENAX failed to show a correlation between FOGQ scores and the volume of the total brain $(P=0.59)$, gray matter $(P=0.68)$ or white matter $(P=0.10)$. Nevertheless, when the diffusion measures averaged across the mean white matter skeleton were assessed, FOGQ scores showed a significant negative correlation with mean FA $(0.38 \pm 0.032 r=-0.49, P=0.026)$, and a significant positive correlation with MD $(0.99 \pm 0.059$ $r=0.50, P=0.022), \mathrm{AD}(1.4 \pm 0.042, r=0.48, P=0.029)$ and RD $(0.79 \pm 0.069, r=0.48, P=0.031$; Table 2$)$.

\section{Local correlation of DTI measures with FOGQ scores}

In the TBSS analysis for the assessment of voxel-byvoxel local correlation, FA showed a significant negative 
Table 1 Clinical assessment of the subjects

\begin{tabular}{llllll}
\hline Subject & Velocity $(\mathbf{m} / \mathbf{s})$ & SL $(\mathbf{m})$ & DST/GC & FOGQ & Presence of FOG \\
\hline 1 & 0.71 & 0.68 & 0.35 & 16 & yes \\
2 & 0.58 & 0.8 & 0.33 & 6 & yes \\
3 & 0.72 & 0.69 & 0.33 & 6 & yes \\
4 & 0.54 & 0.59 & 0.46 & 17 & yes \\
5 & 0.71 & 0.83 & 0.39 & 6 & yes \\
6 & 0.62 & 1.2 & 0.46 & 4 & yes \\
7 & 1.06 & 1.1 & 0.33 & 0 & no \\
8 & 0.96 & 0.66 & 0.31 & 3 & no \\
9 & 0.83 & 0.6 & 0.37 & 8 & no \\
10 & 0.84 & 0.77 & 0.28 & 1 & no \\
11 & 1.1 & 1.2 & 0.25 & 0 & no \\
12 & 1.4 & 0.95 & 0.34 & 0 & no \\
13 & 1.1 & 1 & 0.26 & 1 & no \\
14 & 1.3 & 1.1 & 0.31 & 4 & no \\
15 & 0.99 & 1.0 & 0.27 & 7 & no \\
16 & 1.1 & 1.2 & 0.22 & 1 & no \\
17 & 1.6 & 1.3 & 0.25 & 0 & no \\
18 & 1.4 & 1.3 & 0.20 & 0 & no \\
19 & 1.1 & 1.3 & 0.21 & 0 & no \\
20 & 0.9 & 0.9 & 0.28 & 3 & no \\
\hline 5 & & & 1 & & 0
\end{tabular}

SL: stride length, SW: stride width, DST: double support time, GC: gait cycle, FOGQ: freezing of gait questionnaire, FOG: freezing of gait.

While subjects walked at their own pace repeatedly, the presence of FOG was checked, with special attention being paid to their gait initiation and turning.

Table 2 The behavioral and DTI measures, and their correlations with FOGQ scores

\begin{tabular}{|c|c|c|c|}
\hline \multicolumn{4}{|c|}{ Correlation with FOGQ scores } \\
\hline Parameters & Mean \pm S.D. & $r$ & $P$ \\
\hline$\overline{D S T / G C}$ & $0.31 \pm 0.074$ & 0.63 & $0.003^{*}$ \\
\hline Velocity & $0.98 \pm 0.30$ & -0.79 & $<0.001^{*}$ \\
\hline$S L$ & $0.96 \pm 0.25$ & -0.74 & $<0.001^{*}$ \\
\hline MMSE score & $28 \pm 1.4$ & -0.62 & $0.003^{*}$ \\
\hline \multirow[t]{2}{*}{ FA } & $0.38 \pm 0.032$ & -0.49 & $0.026^{*}$ \\
\hline & $(+0.45 \pm 0.02)$ & & \\
\hline \multirow[t]{2}{*}{ MD } & $0.99 \pm 0.059$ & 0.50 & $0.022^{*}$ \\
\hline & $(† 0.78 \pm 0.03)$ & & \\
\hline \multirow[t]{2}{*}{$A D$} & $1.4 \pm 0.042$ & 0.48 & $0.029^{*}$ \\
\hline & $(† 1.18 \pm 0.03)$ & & \\
\hline \multirow[t]{2}{*}{$\mathrm{RD}$} & $0.79 \pm 0.069$ & 0.48 & $0.031^{*}$ \\
\hline & $(† 0.58 \pm 0.04)$ & & \\
\hline
\end{tabular}

For the DTI measures (FA, MD, AD and RD), values reported by Burzynska et al. using 63 healthy elderly subjects are shown in the parentheses as a reference. $P$-values considered to be significant are followed by an asterisk. DST/GC: double support time/gait cycle, SL: stride length. correlation with the FOGQ scores in three clusters (Figure 2 and Table 3). They were composed of the left superior longitudinal fasciculus (SLF) located close to the premotor area (PM) (Brodmann's area [BA] 6), the right corpus callosum (CC) nearby the anterior cingulate cortex (BA32), and the cerebral peduncle (CP) in the midbrain. A correlation analysis between the FOGQ scores and the MD revealed two significant WM clusters composed of the left posterior limb of the internal capsule (PLIC) located close to the putamen, and of the right cingulum close to the cingulate gyrus (BA24) (Figure 3 and Table 3). In addition, the FOGQ scores showed a significant positive correlation with the AD in the left superior corona radiata (SCR), and with the RD in the left SCR close to the putamen (Figure 3 and Table 3 ).

\section{Multiple linear regression analysis}

To test how much of the variability in the regional diffusion measures was explained by FOG when other parameters were considered, multiple linear regression analyses were performed on the data extracted from the clusters detected by local correlation analysis. In the test of multicollinearity among the independent variables, VIF

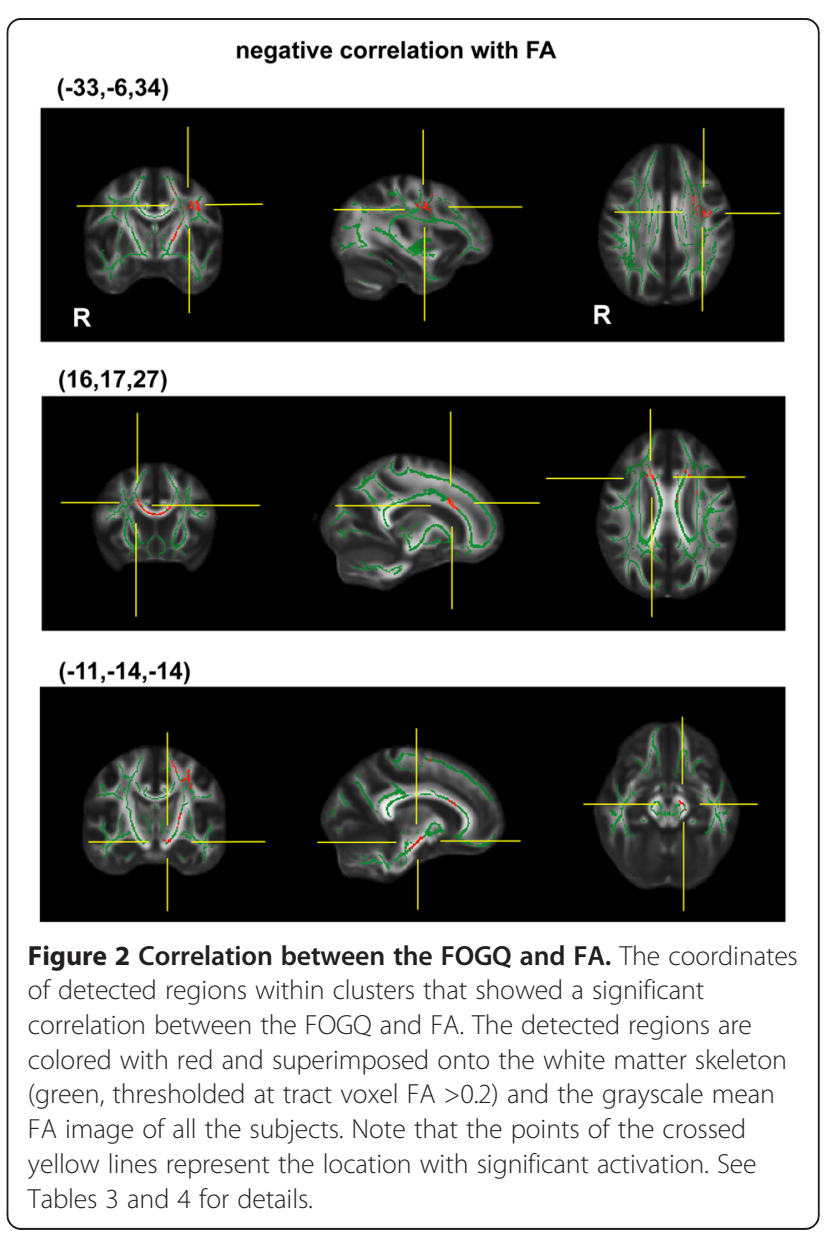


Table 3 The location of each area which showed local correlation between DTI measures and FOGQ scores

\begin{tabular}{|c|c|c|c|c|c|c|c|}
\hline \multirow[t]{2}{*}{ Diffusion measure } & \multicolumn{3}{|c|}{ MNI coordinates } & \multirow[t]{2}{*}{ Cluster size } & \multirow[t]{2}{*}{ Side } & \multirow[t]{2}{*}{ Area } & \multirow[t]{2}{*}{ Nearest GM } \\
\hline & $\mathbf{x}$ & $y$ & z & & & & \\
\hline \multirow[t]{3}{*}{$\overline{F A}$} & -33 & -6 & 34 & 2060 & left & SLF & precentral gyrus (BA6) \\
\hline & 16 & 17 & 27 & 732 & right & CC & anterior cingulate (BA32) \\
\hline & -11 & -14 & -14 & 196 & left & $C P$ & midbrain \\
\hline \multirow[t]{2}{*}{ MD } & -23 & -5 & 18 & 1037 & left & PLIC & putamen \\
\hline & 6 & 18 & 19 & 178 & right & cingulum & anterior cingulate (BA33) \\
\hline$A D$ & -26 & -20 & 29 & 256 & left & SCR & no GM found* \\
\hline $\mathrm{RD}$ & -23 & -5 & 19 & 173 & left & SCR & putamen \\
\hline
\end{tabular}

SLF: superior longitudinal fasciculus, CC: corpus callosum, CP: cerebral peduncle, PLIC: posterior limb of the internal capsule, SCR: superior corona radiata, BA: Brodmann's area, GM: gray matter.

*No gray matter was found around the $11 \times 11 \times 11-\mathrm{mm}^{3}$ cube around this coordinate.

was less than 10 for the FOGQ score (2.8), MMSE score (3.7), SL (7.7), DSP (4.8), cadence (6.8) and SW (2.2) while it was more than 10 for the velocity (25) and GC (22). Since a well-accepted cutoff value of VIF is $10[19,20]$, the walking velocity and GC were excluded from the

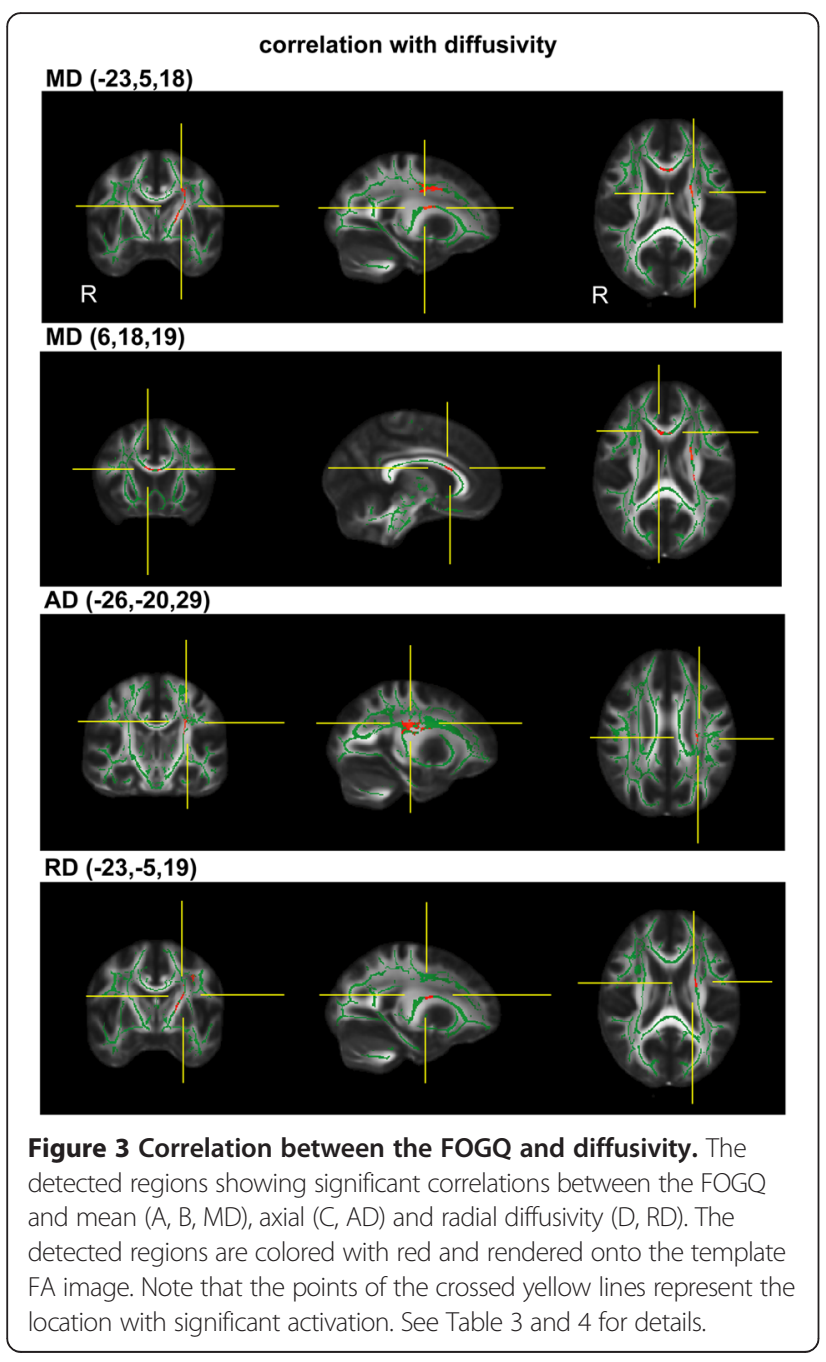

independent variables. In this variable-reduced model, VIF was less than 3 for all the FOGQ (2.6), MMSE scores (1.8), SL (2.8), DSP (2.6), cadence (1.7) and SW (1.8). The adaptability of this reduced multi-regression model was significant for all dependent variables $(P<0.05)$, supporting the appropriateness of the selected independent variables for predicting each dependent variable. In most of the WM regions, only the total FOGQ scores exhibited a significant association $(P<0.05$, Table 4$)$. In the right $C C$, however, both the FOGQ score and the MMSE score exhibited significant associations.

Further, to test the effects of the velocity and GC that were excluded from the reduced multi-regression model, we replaced the FOGQ with the velocity or GC in another series of multiple regression analysis. When the velocity was used, the adaptability of the model was found to be non-significant in all WM clusters, except the right $C C(F=4.06, P=0.016)$. In the right $C C$, only MMSE scores significantly explained the variance in the diffusion-derived variables $(P=0.045)$. When the GC was substituted for the FOGQ scores, the adaptability of the model was non-significant in all WM clusters, except for the right $C C(F=4.05, P=0.016)$. Again in the right $\mathrm{CC}$, only the MMSE scores revealed significant effects $(P=0.012)$.

This analysis suggested that DTI measures of the WM regions, except for the right $\mathrm{CC}$, were specifically correlated with the FOGQ scores, but not with other cognitive or gait parameters.

\section{Discussion}

\section{Assessment of FOG}

The present study revealed a strong correlation between the FOGQ scores and the gait parameters from the computerized gait analysis. PD subjects with FOG have gait disturbance, which result in the abnormality of the gait parameters. For example, FOG affects GC which results in the increase of the stride-to-stride variability [21]. Furthermore, gradual reduction of stride length is associated with 
Table 4 Multilinear regression analysis

\begin{tabular}{|c|c|c|c|c|c|}
\hline Diffusion measure & Area & Variables & Coefficient & $\mathrm{T}$ & $P$ \\
\hline \multirow[t]{6}{*}{$\mathrm{FA}$} & left SLF & FOGQ & -0.81 & -3.7 & $0.003^{*}$ \\
\hline & & MMSE & 0.43 & 2.0 & 0.067 \\
\hline & & SL & -0.42 & -1.8 & 0.092 \\
\hline & & DST/GC & -0.30 & -1.3 & 0.20 \\
\hline & & $\mathrm{Cad}$ & -0.18 & -0.99 & 0.34 \\
\hline & & SW & 0.14 & 0.76 & 0.46 \\
\hline \multirow[t]{6}{*}{ FA } & right CC & FOGQ & -0.57 & -2.7 & $0.018^{*}$ \\
\hline & & MMSE & 0.60 & 3.4 & $0.005^{*}$ \\
\hline & & SL & -0.27 & -1.2 & 0.23 \\
\hline & & DST/GC & 0.02 & 0.093 & 0.93 \\
\hline & & Cad & 0.03 & 0.18 & 0.86 \\
\hline & & SW & -0.18 & -1.0 & 0.32 \\
\hline \multirow[t]{6}{*}{ FA } & left CP & FOGQ & -0.90 & -4.28 & $<0.001^{*}$ \\
\hline & & MMSE & 0.45 & 2.0 & 0.071 \\
\hline & & $S L$ & -0.47 & -2.2 & 0.063 \\
\hline & & DST/GC & -0.08 & -0.38 & 0.71 \\
\hline & & $\mathrm{Cad}$ & -0.18 & -1.1 & 0.30 \\
\hline & & SW & -0.01 & -0.06 & 0.95 \\
\hline \multirow[t]{6}{*}{ MD } & left PLIC & FOGQ & 0.73 & 3.4 & $0.005^{*}$ \\
\hline & & MMSE & -0.37 & -2.1 & 0.07 \\
\hline & & SL & 0.15 & 0.64 & 0.53 \\
\hline & & DST/GC & 0.11 & 0.50 & 0.62 \\
\hline & & Cad & 0.16 & 0.92 & 0.38 \\
\hline & & SW & 0.03 & 0.18 & 0.86 \\
\hline \multirow[t]{6}{*}{ MD } & right cingulum & FOGQ & 0.91 & 4.4 & $<0.001^{*}$ \\
\hline & & MMSE & -0.26 & -1.3 & 0.21 \\
\hline & & SL & 0.17 & 0.81 & 0.44 \\
\hline & & DST/GC & 0.11 & 0.55 & 0.59 \\
\hline & & Cad & 0.04 & 0.26 & 0.80 \\
\hline & & SW & -0.37 & -2.1 & 0.08 \\
\hline \multirow[t]{6}{*}{$A D$} & left SCR & FOGQ & 0.96 & 3.7 & $0.003^{*}$ \\
\hline & & MMSE & 0.05 & 0.22 & 0.83 \\
\hline & & SL & 0.21 & 0.76 & 0.46 \\
\hline & & DST/GC & -0.17 & -0.63 & 0.54 \\
\hline & & Cad & -0.06 & -0.3 & 0.77 \\
\hline & & SW & 0.14 & 0.65 & 0.53 \\
\hline \multirow[t]{6}{*}{$\mathrm{RD}$} & left SCR & FOGQ & 0.69 & 2.7 & $0.019^{*}$ \\
\hline & & MMSE & -0.08 & -0.39 & 0.71 \\
\hline & & SL & -0.06 & -0.21 & 0.84 \\
\hline & & DST/GC & 0.12 & 0.48 & 0.64 \\
\hline & & $\mathrm{Cad}$ & 0.02 & 0.11 & 0.91 \\
\hline & & SW & -0.07 & -0.32 & 0.75 \\
\hline
\end{tabular}

The table shows the results of multilinear regression analysis of all those six independent variables using average activation of each area in which significant local correlation between FOGQ and FA was found as a dependent variable. The significant $P$-values are noted by an asterisk.

FOGQ: Freezing of Gait Questionnaire, MMSE: Mini-Mental State Examination, SL: stride length, DST/GC: double support time/gait cycle, cad: cadence, SW: stride width. 
FOG [22]. The result from the present study concurs with these findings, although we should be careful not to simply equate FOG and gait disturbance. That is, although most subjects with FOG show profound gait disturbance, some subjects with FOG may show normal gait parameters upon a gait analysis, reflecting fluctuating nature of FOG [6].

Nevertheless, the multiple regression analysis indicated that only the FOGQ score exhibited a significant effect as an independent variable explaining changes of the DTI measures in almost all the WM clusters detected in the local correlation analysis. The multiple regression analysis substituting the FOGQ scores for the walking velocity or GC failed to produce significant results in the model in most of the WM clusters. These results indicate that the diffusion measures in the WM clusters (except for $\mathrm{CC}$ ) were strongly correlated with the severity of FOG specifically, not with cognitive decline or gait disturbance in general.

\section{Interhemispheric connections}

In the CC, FA exhibited a significant negative correlation with FOGQ and MMSE scores. WM integrity of the CC is significantly correlated with quantitative measures of gait in the elderly population [9]. It has been suggested that the CC may transfer sensory information necessary for gait planning interhemispherically [23]. Moreover, a recent study demonstrated that perceptual judgment of an upcoming doorway is more strongly affected in patients with FOG than those without [24]. These findings suggest that CC may affect the ability of gait planning according to sensory information in the environment which might be related to FOG.

\section{Corticofugal and thalamocortical fibers}

The present study revealed a negative correlation between FOGQ scores and FA in the ventromedial part of the $\mathrm{CP}$ at the midbrain level. The $\mathrm{CP}$ includes the corticofugal tract, which connects the brainstem and the spinal cord with cortical motor areas including the primary motor cortex, supplementary motor area (SMA) and PM [25]. The CP may also include the fibers projecting to the pedunculopontine nucleus (PPN) and/or the mesencephalic locomotor region (MLR). The neural circuits connecting the PPN, basal ganglia, motor cortex and limbic system are hypothesized to be responsible for FOG [26]. Moreover, recent study revealed that the PD patients with FOG showed more increase of brain activity in the PPN/MLR during imagery of walking compared with those without FOG [27]. Taken together, the disruption of the projecting fibers to PPN/MLR at the level of $\mathrm{CP}$ appears to be responsible for FOG.

There was a positive correlation between MD in the PLIC and FOGQ scores. Damage to the PLIC encompassing parts of the corticofugal tracts could result in poor motor outcomes [28]. In infants with low birth weight, reduced FA in the PLIC has been shown to be correlated with the severity of gait deficits in the future [29].

Both $\mathrm{AD}$ and $\mathrm{RD}$ were significantly correlated with the FOGQ scores in the left SCR. Hence, the damage to both myelin and axons might contribute to the WM damage seen here. Damage of the thalamocortical sensory tracts at the SCR can cause both sensory deficits and gait disturbance [30]. Considering that FOG can be ameliorated or exacerbated by sensory stimuli [31], sensorimotor networks are likely to be involved.

\section{Fronto-parietal connections and cingulum}

It has been suggested that FOG may result from dysfunction of the PM-parietal circuits [32]. Consistent with this view, we found a significant negative correlation between FA and FOGQ scores in the SLF close to the PM. The SLF is likely to contribute to visuospatial processing [33] which might be related to FOG.

The cingulum collects projections from the cingulate cortex and extends into the temporal or frontal lobe [34]. In the present study, MD in the cingulum exhibited a positive correlation with FOGQ, consistent with the hypothesis that a local network in SMA and adjacent cingulate cortex might be responsible for FOG [35]. The cingulate regions are activated during gait movement $[10,36]$ and are also thought to be involved in the planning of gait $[37,38]$. The cingulum bundle participates in the limbic circuit, which is thought to be involved in FOG together with the motor and cognitive circuits in patients with PD [26]. Based on these considerations, the present findings in the cingulum might reflect not only the motor but also the emotional aspects of FOG.

\section{Brain volume, global diffusion measures and FOG}

Previous studies indicate that gray matter atrophy is related to gait impairment [39], as well as the volume of WM lesions [40]. However, our analysis failed to reveal a significant correlation between WM atrophy and FOGQ, whereas all of the diffusion measures were significantly correlated with FOGQ. Thus, it appears that the diffusion measures were more sensitive than WM atrophy in patients with ARWMC.

We did not take the WM lesion volume into account in the TBSS analysis since we focused on the integrity of the white matter. However, we could not exclude a possibility that such WM volume changes might affect the TBSS results.

\section{Proposed mechanisms underlying FOG}

The present findings raise the question of how the WM abnormalities identified above (CC, PLIC, SCR, CP, and SLF) might explain FOG. Youn et al. [41] performed a region-of-interest (ROI) analysis on DTI data and found 
that the FA values of the bilateral PPN, bilateral PM, right orbitofrontal area and left SMA were lower in the ARWMC patients with FOG compared to those without. Their finding partly concurs the present result, which showed that the FA in the SLF close to the PM, and also in the CP close to the midbrain PPN, was correlated with FOG. The areas detected slightly differed probably because of the difference in the methodology, especially the spatial normalization procedure (TBSS versus ROI analysis). The present result suggests that multiple neural substrates related to the control of walking may be involved in FOG in consistent with the study by Youn et al. [41]. This notion favors the view that FOG is likely to be a clinical condition resulting from multiple pathophysiological issues involving one or more nodes of the neural network regulating walking behavior. Since visual stimuli or some other extra cues can help or exacerbate FOG [31], the phenomenon might emerge when extra demands for sensory, cognitive, or emotional processing overburden the damaged neural substrates.

\section{Limitations of this study}

We enrolled patients who were regularly visiting a neurology outpatient clinic. Thus, the population might be different from those who were diagnosed as having ARWMC in radiology departments or in general elderly population. Therefore, a further study will be required to test if the present finding can be generalized to those population.

In the present study, we focused on the relationship between WM abnormality and FOG. We employed a relatively small number of subjects to perform a comprehensive investigation. Since there is no established objective measure of FOG, we avoided classifying subjects with FOG and those without, and used FOGQ as a parameter for a correlation analysis instead. Because of this strategy, we have included the subjects who did not clearly present FOG, which is often invisible at the physician's room. To reduce ambiguity regarding the subject selection, we did our best to discriminate FOG and general gait disturbance in the multiple regression analysis, but it would still be difficult to discuss the mechanisms of FOG completely segregated from those of gait disturbance in general.

In the present study, we used the $\mathrm{T}_{2}$-weighted images only for the purpose of subject recruitment, but did not analyze these non-quantitative data. We reviewed the individual $\mathrm{T}_{2}$-weighted images of each subject, and visually investigated if the regions detected in the DTI analysis corresponded to ARWMC regions in the $\mathrm{T}_{2}$-weighted images. In most of the subjects, ARWMC were present in all the detected regions except for the CP. This result is reasonable since we tested the correlation between the FOGQ scores and the diffusion parameters, which should reflect different aspects of anatomical structure seen in the $\mathrm{T}_{2}$-weighted images. For example, as minor reduction of white matter integrity resulting from remote ischemic lesions would be captured by DTI, but not necessarily by $\mathrm{T}_{2}$-weighted images. Future research comparing DTI and quantitative $\mathrm{T}_{2}$-weighted images is awaited. We discussed FOG mainly in relation to the underlying pathophysiology of FOG in PD. The symptoms of FOG seem very similar across different diseases such as PD and other types of Parkinsonism. However, it remains unclear whether the mechanisms underlying FOG are shared across related diseases. Therefore, caution must be exercised in generalizing the mechanisms of FOG suggested here. Future studies are necessary to compare the mechanisms of FOG across different diseases.

\section{Conclusion}

While disconnections in the brain have previously been suggested as a cause of FOG, few studies have directly addressed this issue. To our knowledge, the present study provides the first experimental evidence that FOG is related to WM disruption, with the current results revealing that WM damage in the SLF, CC, PLIC, cingulum, CR and CP was correlated with FOG.

\section{Abbreviations}

ARWMC: Age related white matter change; FA: Fractional anisotropy; FOG: Freezing of gait; PD: Parkinson's disease.

\section{Competing interests}

The authors declare that they have no competing interests.

\section{Authors' contributions}

$\mathrm{Kl}$ designed and conducted the study, collected data, drafted and revised the paper. He also has full access to all the data. He is a guarantor. HF designed and supervised the study, and revised the paper with intellectual suggestions. NO conducted analyses and revised the paper with intellectual suggestions. HT recruited the subjects, served for the interpretation of the data, and revised the manuscript with intellectual suggestions. YO recruited the subjects, served for the interpretation of the data, and revised the manuscript with intellectual suggestions. MN served for the interpretation of the data, and revised the manuscript with intellectual suggestions. DB served for the interpretation of the data, and revised the manuscript with intellectual suggestions. MH served for the interpretation of the data, and revised the manuscript with intellectual suggestions. TH designed and supervised the study, and revised the manuscript with intellectual suggestions. All the authors listed approved for the present paper to be published.

\section{Funding}

The research was partly supported by grants from KAKENHI (24500573) and the Sumitomo Life Social Welfare Services Foundation to K.I., and by grants from Japanese Society in the Promotion of Science (21613004) and Global COE Program to N.O., and by grants from KAKENHI (26120008) and Development of BMI Technologies for Clinical Application carried out under the Strategic Research Program for Brain Sciences to T.H. The NINDS Intramural Program provided additional support.

\section{Author details}

'Human Brain Research Center, Kyoto University Graduate School of Medicine, 54 Kawahara-cho, Shogoin, Sakyo-ku, Kyoto 606-8507, Japan. ${ }^{2}$ Human Motor Control Section, Medical Neurology Branch, National Institute of Neurological Disorders and Stroke, National Institutes of Health, Bethesda, MD, USA. ${ }^{3}$ Department of Behavioral Neurology and Cognitive Neuroscience, Tohoku University, Graduate School of Medicine, Sendai, Miyagi, Japan. 
${ }^{4}$ Department of Neurology, Sakakibara-Hakuho Hospital, Tsu, Mie, Japan. ${ }^{5}$ Department of Neurology, Mie University, Graduate School of Medicine, Tsu, Mie, Japan. ${ }^{6}$ Department of Physical Therapy, Kyoto University Hospital, Kyoto, Japan. ${ }^{7}$ Department of Advanced Neuroimaging, Integrative Brain Imaging Center, National Center of Neurology and Psychiatry, Kodaira, Japan. ${ }^{8}$ PRESTO, JST, Kawaguchi, Saitama, Japan.

Received: 2 July 2014 Accepted: 17 October 2014

Published online: 20 January 2015

\section{References}

1. Giladi N, Nieuwboer A: Understanding and treating freezing of gait in parkinsonism, proposed working definition, and setting the stage. Mov Disord 2008, 23(Suppl 2):S423-S425.

2. Factor SA: The clinical spectrum of freezing of gait in atypical parkinsonism. Mov Disord 2008, 23(Suppl 2):S431-S438.

3. de Leeuw FE, de Groot JC, Achten E, Oudkerk M, Ramos LM, Heijboer R, Hofman A, Jolles J, van Gijn J, Breteler MM: Prevalence of cerebral white matter lesions in elderly people: a population based magnetic resonance imaging study: the Rotterdam Scan Study. J Neurol Neurosurg Psychiatry 2001, 70:9-14.

4. Hachinski VC, Potter P, Merskey H: Leuko-araiosis. Arch Neurol 1987, 44:21-23.

5. Inzitari M, Pozzi C, Rinaldi LA, Masotti G, Marchionni N, Di Bari M: Cognitive and functional impairment in hypertensive brain microangiopathy. J Neurol Sci 2007, 257:166-173.

6. Nutt JG, Bloem BR, Giladi N, Hallett M, Horak FB, Nieuwboer A: Freezing of gait: moving forward on a mysterious clinical phenomenon. Lancet Neurol 2011, 10:734-744.

7. Giladi N, Shabtai H, Simon ES, Biran S, Tal J, Korczyn AD: Construction of freezing of gait questionnaire for patients with Parkinsonism. Parkinsonism Relat Disord 2000, 6:165-170.

8. Charlton RA, Barrick TR, Mclntyre DJ, Shen Y, O'Sullivan M, Howe FA, Clark CA, Morris RG, Markus HS: White matter damage on diffusion tensor imaging correlates with age-related cognitive decline. Neurology 2006, 66:217-222.

9. Bhadelia RA, Price LL, Tedesco KL, Scott T, Qiu WQ, Patz S, Folstein M, Rosenberg I, Caplan LR, Bergethon P: Diffusion tensor imaging, white matter lesions, the corpus callosum, and gait in the elderly. Stroke 2009, 40:3816-3820

10. Iseki K, Hanakawa T, Hashikawa K, Tomimoto H, Nankaku M, Yamauchi H, Hallett M, Fukuyama H: Gait disturbance associated with white matter changes: a gait analysis and blood flow study. Neuroimage 2010, 49:1659-1666.

11. Smith SM, Jenkinson M, Johansen-Berg H, Rueckert D, Nichols TE, Mackay CE, Watkins KE, Ciccarelli O, Cader MZ, Matthews PM, Behrens TE: Tract-based spatial statistics: voxelwise analysis of multi-subject diffusion data. Neuroimage 2006, 31:1487-1505.

12. Fazekas F, Chawluk JB, Alavi A, Hurtig HI, Zimmerman RA: MR signal abnormalities at $1.5 \mathrm{~T}$ in Alzheimer's dementia and normal aging. AJR Am J Roentgenol 1987, 149:351-356.

13. Song SK, Sun SW, Ramsbottom MJ, Chang C, Russell J, Cross AH: Dysmyelination revealed through MRI as increased radial (but unchanged axial) diffusion of water. Neuroimage 2002, 17:1429-1436

14. Budde MD, Kim JH, Liang HF, Schmidt RE, Russell JH, Cross AH, Song SK: Toward accurate diagnosis of white matter pathology using diffusion tensor imaging. Magn Reson Med 2007, 57:688-695.

15. Song SK, Sun SW, Ju WK, Lin SJ, Cross AH, Neufeld AH: Diffusion tensor imaging detects and differentiates axon and myelin degeneration in mouse optic nerve after retinal ischemia. Neuroimage 2003, 20:1714-1722.

16. Bennett IJ, Madden DJ, Vaidya CJ, Howard DV, Howard JH Jr: Age-related differences in multiple measures of white matter integrity: A diffusion tensor imaging study of healthy aging. Hum Brain Mapp 2010, 31:378-390.

17. Smith SM, Zhang Y, Jenkinson M, Chen J, Matthews PM, Federico A, De Stefano N: Accurate, robust, and automated longitudinal and cross-sectional brain change analysis. Neuroimage 2002, 17:479-489.

18. Smith SM, Nichols TE: Threshold-free cluster enhancement: addressing problems of smoothing, threshold dependence and localisation in cluster inference. Neuroimage 2009, 44:83-98.

19. Chatterjee S, Hadi A: In Regression Analysis by Examples. 4th edition. Edited by Balding DJ. New York: John Wiley \& Sons; 2006.

20. Freund R, Wilson W, Ping S: Regression analysis. In Statistical Modeling of a Response Variable. Edited by Singer T. San Diego: Academic; 2006.
21. Hausdorff JM, Schaafsma JD, Balash Y, Bartels AL, Gurevich T, Giladi N: Impaired regulation of stride variability in Parkinson's disease subjects with freezing of gait. Exp Brain Res 2003, 149:187-194.

22. Chee R, Murphy A, Danoudis M, Georgiou-Karistianis N, lansek R: Gait freezing in Parkinson's disease and the stride length sequence effect interaction. Brain 2009, 132:2151-2160.

23. Park HJ, Kim JJ, Lee SK, Seok JH, Chun J, Kim DI, Lee JD: Corpus callosal connection mapping using cortical gray matter parcellation and DT-MRI. Hum Brain Mapp 2008, 29:503-516.

24. Almeida QJ, Lebold CA: Freezing of gait in Parkinson's disease: a perceptual cause for a motor impairment? I Neurol Neurosurg Psychiatry 2010, 81:513-518.

25. Newton JM, Ward NS, Parker GJ, Deichmann R, Alexander DC, Friston KJ, Frackowiak RS: Non-invasive mapping of corticofugal fibres from multiple motor areas-relevance to stroke recovery. Brain 2006, 129:1844-1858.

26. Lewis SJ, Barker RA: A pathophysiological model of freezing of gait in Parkinson's disease. Parkinsonism Relat Disord 2009, 15:333-338.

27. Snijders AH, Leunissen I, Bakker M, Overeem S, Helmich RC, Bloem BR, Toni I: Gait-related cerebral alterations in patients with Parkinson's disease with freezing of gait. Brain 2011, 134:59-72.

28. Jang SH: A review of corticospinal tract location at corona radiata and posterior limb of the internal capsule in human brain. NeuroRehabilitation 2009, 24:279-283.

29. Rose J, Mirmiran M, Butler EE, Lin CY, Barnes PD, Kermoian R, Stevenson DK: Neonatal microstructural development of the internal capsule on diffusion tensor imaging correlates with severity of gait and motor deficits. Dev Med Child Neurol 2007, 49:745-750.

30. Shinoura N, Suzuki Y, Yoshida M, Yamada R, Tabei Y, Saito K, Yagi K: Assessment of the corona radiata sensory tract using awake surgery and tractography. J Clin Neurosci 2009, 16:764-770.

31. Hallett M: The intrinsic and extrinsic aspects of freezing of gait. Mov Disord 2008, 23(Suppl 2):S439-S443.

32. Bartels $A L$, Leenders $\mathrm{KL}$ : Brain imaging in patients with freezing of gait Mov Disord 2008, 23(Suppl 2):S461-S467.

33. Hoeft F, Barnea-Goraly N, Haas BW, Golarai G, Ng D, Mills D, Korenberg J, Bellugi U, Galaburda A, Reiss AL: More is not always better: increased fractional anisotropy of superior longitudinal fasciculus associated with poor visuospatial abilities in Williams syndrome. J Neurosci 2007, 27:11960-11965.

34. Schmahmann JD, Pandya DN, Wang R, Dai G, D'Arceuil HE, de Crespigny AJ, Wedeen VJ: Association fibre pathways of the brain: parallel observations from diffusion spectrum imaging and autoradiography. Brain 2007, 130:630-653.

35. Hashimoto T: Speculation on the responsible sites and pathophysiology of freezing of gait. Parkinsonism Relat Disord 2006, 12:S55-S62.

36. Hanakawa T, Fukuyama H, Katsumi Y, Honda M, Shibasaki H: Enhanced lateral premotor activity during paradoxical gait in Parkinson's disease. Ann Neurol 1999, 45:329-336.

37. Dobkin BH, Firestine A, West M, Saremi K, Woods R: Ankle dorsiflexion as an $\mathrm{fMRI}$ paradigm to assay motor control for walking during rehabilitation. Neuroimage 2004, 23:370-381.

38. Iseki K, Hanakawa T, Shinozaki J, Nankaku M, Fukuyama H: Neural mechanisms involved in mental imagery and observation of gait. Neuroimage 2008, 41:1021-1031.

39. Rosano C, Sigurdsson S, Siggeirsdottir K, Phillips CL, Garcia M, Jonsson PV, Eiriksdottir G, Newman AB, Harris TB, van Buchem MA, Gudnason V, Launer LJ: Magnetization transfer imaging, white matter hyperintensities, brain atrophy and slower gait in older men and women. Neurobiol Aging 2010, 31:1197-1204.

40. Soumare A, Elbaz A, Zhu Y, Maillard P, Crivello F, Tavernier B, Dufouil C, Mazoyer B, Tzourio C: White matter lesions volume and motor performances in the elderly. Ann Neurol 2009, 65:706-715.

41. Youn J, Cho JW, Lee WY, Kim GM, Kim ST, Kim HT: Diffusion tensor imaging of freezing of gait in patients with white matter changes. Mov Disord 2012, 27:760-764. 